\title{
Isolation and Characterization of Organosolv and Alkaline Lignins from Hardwood and
}

\section{Softwood Biomass}

Christos Nitsos ${ }^{1}$, Ryan Stoklosa ${ }^{1,2}$, Anthi Karnaouri ${ }^{1,3}$, Dimitrij Vörös ${ }^{1}$, Heiko Lange ${ }^{3}$, David Hodge ${ }^{1,2}$, Claudia Crestini ${ }^{3}$, Ulrika Rova ${ }^{1}$, Paul Christakopoulos ${ }^{1 *}$

${ }^{1}$ Biochemical Process Engineering, Division of Chemical Engineering, Department of Civil,

Environmental and Natural Resources Engineering, Luleå University of Technology, 971-87 Luleå,

Sweden

${ }^{2}$ Department of Chemical Engineering and Materials Science, Michigan State University

${ }^{3}$ University of Rome 'Tor Vergata', Department of Chemical Sciences and Technologies,

Via della Ricerca Scientifica, 00133 Rome, Italy

*corresponding author, e-mail: paul.christakopoulos@ltu.se

\section{Supporting Information}

No of pages: 3

No of Figures: 1

No of Tables: 1 


\section{Analysis and characterization procedure}

The methodology followed in this work for the analysis and characterization of all the liquid (pretreatment liquor) and solid (alkaline and organosolv pretreated biomass samples, isolated lignin samples) samples, is described in detail in the Experimental section of the manuscript, and is also schematically represented in Figure S1-Sn.

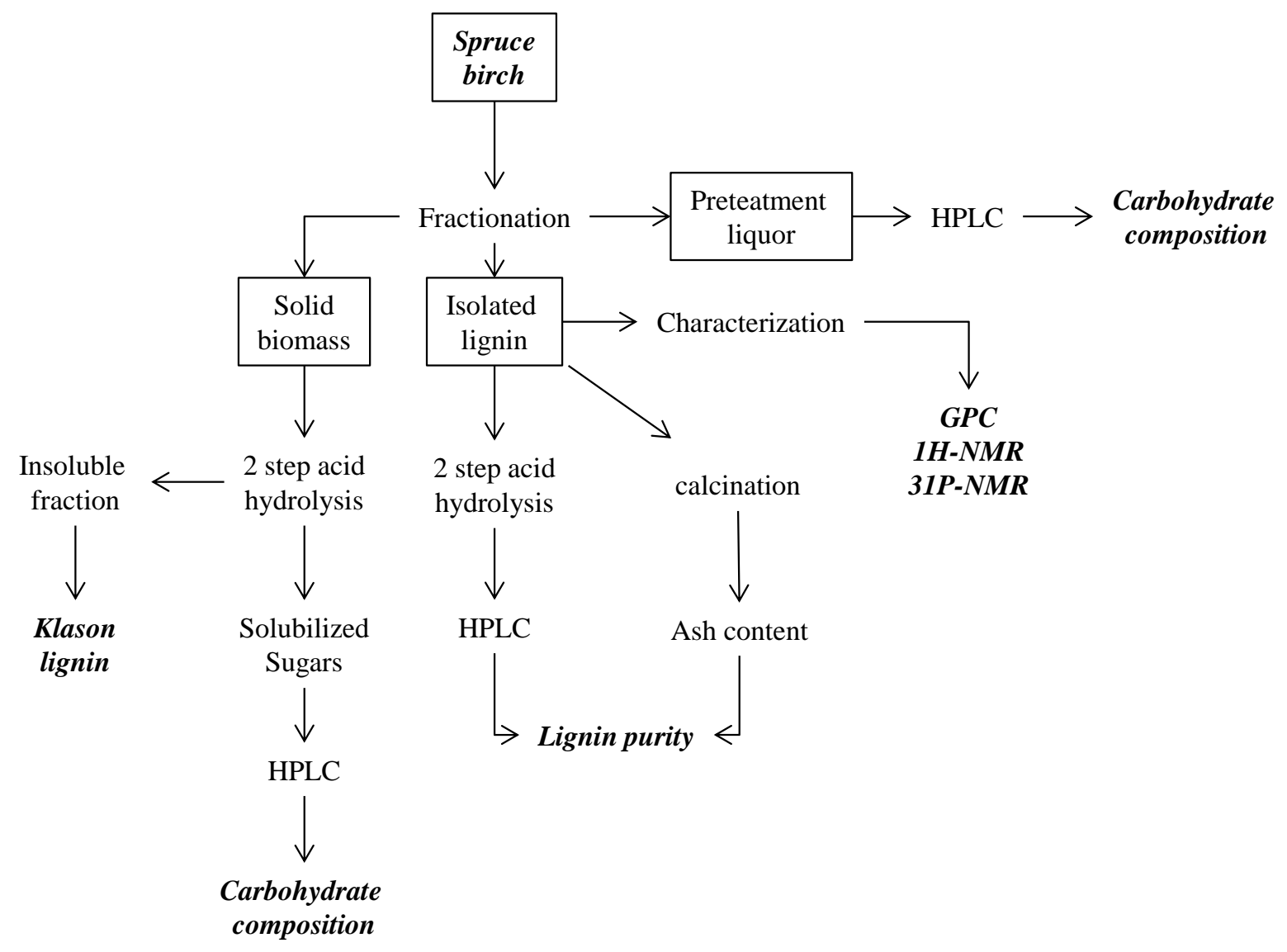

Figure S1-Sn. Outline of analysis and characterization experiments of samples

\section{Composition of organosolv pretreatment liquor}

The chemical composition of the organosolv pretreatment liquors from spruce and birch biomass after the isolation of lignin is presented in Table S1-Sn. The results were obtained by HPLC analysis of the liquors and besides the concentrations of cellulosic and hemicellulosic sugars, the concentrations of the main degradation producs/inhibitors suchas organic acids and furans is also reported. 
Table S1-Sn. Chemical composition of organosolv pretreatment liquor from spruce and birch biomass samples

\begin{tabular}{|c|c|c|c|c|c|c|c|c|c|c|c|c|}
\hline \multirow{4}{*}{$\begin{array}{l}\text { Time } \\
\text { Particle size } \\
\mathrm{H}_{2} \mathrm{SO}_{4}(\mathrm{w} / \mathrm{v})\end{array}$} & \multicolumn{8}{|c|}{ Biomass extracted with $50 \%(v / v)$ ethanol } & \multirow{2}{*}{\multicolumn{4}{|c|}{$\begin{array}{c}\text { Biomass extracted with } 60 \%(\mathrm{v} / \mathrm{v}) \text { ethanol } \\
60 \mathrm{~min}\end{array}$}} \\
\hline & \multicolumn{4}{|c|}{$60 \mathrm{~min}$} & \multicolumn{4}{|c|}{$103 \mathrm{~min}$} & & & & \\
\hline & & & & & & & & & & & \multicolumn{2}{|c|}{$4 \mathrm{~mm}$} \\
\hline & $0 \%$ & $1 \%$ & $0 \%$ & $1 \%$ & $0 \%$ & $1 \%$ & $0 \%$ & $1 \%$ & $0 \%$ & $1 \%$ & $0 \%$ & $1 \%$ \\
\hline \multicolumn{13}{|c|}{ Spruce biomass } \\
\hline Glucose & 0.36 & 2.80 & 0.45 & 2.33 & - & 2.44 & - & 2.41 & 0.02 & 2.59 & 0.02 & 2.61 \\
\hline Xylose & - & - & - & - & - & - & - & - & - & - & - & - \\
\hline Galactose & 0.71 & 3.65 & 0.81 & 2.97 & 1.21 & 2.52 & 1.37 & 1.42 & 0.21 & 2.51 & 0.36 & 2.44 \\
\hline Mannose & 0.08 & 1.68 & 0.13 & 1.41 & - & 1.07 & - & - & 0.21 & 2.03 & 0.07 & 2.12 \\
\hline Arabinose & - & - & - & - & - & - & - & - & & & & \\
\hline Acetic acid & 0.14 & 0.45 & 0.14 & 0.39 & - & 0.47 & 0.20 & 0.48 & 0.12 & 0.36 & 0.13 & 0.30 \\
\hline Formic acid & 0.15 & 0.38 & 0.19 & 0.33 & - & 0.35 & 0.15 & 0.07 & 0 & 0.33 & 0.07 & 0.32 \\
\hline HMF & - & 0.86 & 0.01 & 0.73 & 0.15 & 0.60 & 0.15 & 0.72 & - & 0.72 & - & 0.59 \\
\hline Furfural & - & 0.77 & - & 0.63 & - & 0.05 & - & 0.68 & - & 0.71 & - & 0.62 \\
\hline \multicolumn{13}{|c|}{ Birch biomass } \\
\hline Glucose & - & 1.63 & - & 1.57 & - & 1.34 & 0.78 & 2.06 & - & 1.54 & 0.45 & 1.48 \\
\hline Xylose & 0.38 & 2.86 & 0.65 & 3.26 & 0.66 & 2.01 & 0.99 & 2.78 & 0.31 & 3.23 & - & 2.95 \\
\hline Galactose & - & - & - & - & - & - & - & - & - & - & - & - \\
\hline Mannose & - & - & - & - & - & - & - & - & - & - & - & - \\
\hline Arabinose & 0.54 & 1.35 & 1.21 & 1.47 & 0.60 & 0.56 & - & 1.65 & 0.75 & 1.69 & 1.35 & 1.75 \\
\hline Acetic acid & 0.43 & 1.07 & 0.46 & 0.98 & 0.12 & 1.12 & 0.41 & 1.19 & 0.01 & 0.8 & - & 0.81 \\
\hline Formic acid & - & 2.14 & 0.02 & 2.32 & - & 1.37 & - & 0.57 & 0.05 & 2.56 & - & 0.97 \\
\hline HMF & - & - & - & - & - & 0.04 & - & 0.03 & - & - & - & - \\
\hline Furfural & - & 0.23 & - & 0.15 & - & 0.18 & - & 0.54 & - & 0.17 & - & 1.30 \\
\hline
\end{tabular}

